unaltered. This is the remarkable result, that the force is flavour independent, which is expected if the strong forces are indeed due to the exchange of coloured gluons as embodied in QCD.

These narrow states, too low in mass to decay to $b \bar{q}+\overline{b q}$ mesons, are further expected in QCD to decay by the emission of three gluons (the smallest number allowed by a variety of selection rules). Detailed analysis of the hadronic final state of the $\Upsilon$ indicates that even this prediction is consistent with all of the observations. Thus this $\Upsilon$ system confirms dramatically the QCD model of strong interactions.

The $\Upsilon$ "' should be a prolific source of $B$ mesons, and hence detailed studies of their properties (and those of the $b$ quark) will be possible. A B meson has yet to be demonstrated at Cornell (an earlier possibility from a CERN experiment has since disappeared with further data). Its decay modes are many and the multiplicity of particles in each is expected to be very high, making a clean identification difficult. Nonetheless, it is possible to look for irregularities that might be associated with the "' and hence the B particles. An excess of $\mathrm{K}$ mesons is observed and these are expected if the $\mathrm{b}$ quark (within the $\mathrm{B}$ meson) undergoes a weak decay transition to the charm quark, which has a well documented prediliction for strange particles in its decay. The excess is, within the large errors, just what would be expected. Furthermore, the semi-leptonic weak decays of the $\mathrm{B}$ mesons $(\mathrm{B} \rightarrow x \mathrm{x} v$, where $v$ represents one or more hadrons) lead to increased numbers of electrons and muons. Such increases are observed and the inferred branching ratios for these decays are $24 \pm 6$ per cent, whereas the standard model, containing a top quark, would suggest a value of $\sim 34$ per cent, not widely inconsistent with the experimental value. Thus the data, sparse as they are at the moment, suggest that the standard model remains intact with the most likely existence of a top (charge 2/3) quark and a weak decay chain $t \rightarrow b \rightarrow c \rightarrow s$. Higher energies than those achieved so far at PETRA ( $38.6 \mathrm{GeV}$ ) will be needed in the search for this quark. A very recent paper (S. Glashow Phys. Rev. Lett. 45, 1914; 1980) puts forward further theoretical arguments for a mass of $38+2 \mathrm{GeV}$ for the top quark. Experimentalists should soon be able to check on this. The present gauge model relies on its existence. The new facilities in Cornell will undoubtedly produce far more quantitative results in the future. These will allow more systematic tests of our current ideas of the quarks and their strong and weak interactions and perhaps in this quantitative exposure new possibilities will emerge. After all, we have as yet no explanation for the number of generations of quarks and leptons. Experiments will perhaps one day provide the clue. An exciting few years are in store at Cornell.

\section{Molecular hydrogen in T Tauri}

\section{from Stephen P. Maran}

THE discovery of ultraviolet molecular hydrogen emission in $T$ Tauri with the International Ultraviolet Explorer satellite (see Brown et al. this issue of Nature p.34) has considerable potential for three fields of research: the nature of $\mathrm{T}$ Tauri stars and their associated nebulae; the formation and development of the Solar System; and the study of molecular hydrogen in the Galaxy. T Tauri stars have masses about equal to that of the Sun, but are rotating much more rapidly and are thought to be stars still-in-the-making, only about a million years old and still contracting towards the Main Sequence. Some appear to be losing mass, the so-called ' $T$ Tauri wind', while others appear to be still accreting matter from the surrounding nebulae. It is widely accepted that the Sun passed through a T Tauri phase and models of Solar System formation take into account the postulated high wind and luminosity and the concomitant decreasing gravitational attraction of the early Sun to solve a number of problems. Among them are the placing of Mercury and perhaps other planets into their present orbits, the sweeping out of excess gas and dust in the protoplanetary nebula, the dispersal (in modern versions of the Laplace nebular hypothesis) of gas rings in the planetary orbits, the formation of chondrules found in meteorites, and the reduction of the Sun's rotation to its present very low rate. $\mathrm{T}$ Tau itself, the prototype of the stellar class, is associated with a small luminous cloud called Burnham's nebula.

It had been thought unlikely that ultraviolet emission known to occur in nebulae would be detectable in Burnham's nebula with instruments having the sensitivities of the two IUE spectrographs. Brown et al. confirmed this, for they found no previously known nebular lines in the spectrum of the object, but instead discovered emission in the $\mathrm{H}_{2}$ Lyman bands that previously were seen only in sunspots. In recent years, astronomers have concluded that the interstellar gas is not dominated simply by atomic hydrogen but that there is also a substantial molecular component. This has been largely inferred from observations of the $\mathrm{CO}$ molecule in the galactic plane but $\mathrm{H}_{2}$ in the general medium has also been seen through its ultraviolet absorption lines (a technique feasible only in the directions of appropriate hot stars that serve as background continuum sources), and $\mathrm{H}_{2}$ in several nebulae (including Burnham's nebula) has been found via the infrared

Stephen P. Maran is Senior Staff Scientist in the Laboratory for Astronomy and Solar Physics, NASA-Goddard Space Flight Center, Maryland. vibration bands. More recently, a boundfree absorption continuum of the $\mathrm{H}_{2}{ }^{+}$ion has been detected in planetary nebulae $(\mathrm{S}$. Heap and T.P. Stecher Proc. Conf. on the Universe at Ultraviolet Wavelengths 1980).

Brown et al. distinguish between two mechanisms for $\mathrm{H}_{2}$ excitation in Burnham's nebula and favour the 'shock excitation' model. The nebula is much smaller than the Oort cometary cloud that surrounds the Sun, and hence phenomena on its scale may well be relevant to the early evolution of the Solar System. Since $\mathrm{H}_{2}$ must be a principal component of the nebula, observations with more sensitive ultraviolet instruments having very fine angular resolution (notably, the $\mathrm{High}$ Resolution Spectrograph now under development for flight on the Space Telescope) will enable astronomers to investigate the dynamics of the shocked gas. In the meantime, nebular specialists will be busy calculating where else in the Galaxy this new diagnostic may be detectable.

\section{Planarian neoblasts}

\section{from J. Baguna}

IN a News and Views article on 21 August 1980 Jonathan Slack' reviewed briefly the sources of the cells involved in regeneration in planaria and, in doing so, made several assertions that deserve comment.

(1) 'Neoblasts' are indeed small basophilic cells present in planaria. But to think of them as cells 'reserved' for regeneration is equivocal since many planarian species, having sexual reproduction, never regenerate in nature. So what are the neoblasts for? The answer lies in considering the neoblast not just as a 'reserve' cell but as the stem cell of most differentiated cell types supplying transit cells to balance the death of cells.

(2) In planarian regeneration it is meaningless to argue whether neoblasts come from all parts of the body or from local tissues through metaplasia because the blastema can be formed (and is formed) from pre-existing local neoblasts proliferating near the wound. Moreover, Wolff's experiments ${ }^{2}$, which have been cited as evidence for migratory neoblasts, have been consistently criticized $^{3 \cdot 6}$ because at most, they only show the 'repopulation' of the irradiated region by healthy neoblasts through cell division and do not prove a direct migration of neoblasts from unirradiated regions to the wound.

(3) Slack points out that "histological and electron microscopic studies suggest that local dedifferentiation does occur'. The

$J$ Baguna is in the Department of Genetics, University of Barcelona, Spain. 